\title{
NOTES
}

\section{Photopolymerization of Methyl Methacrylate with $\mathrm{N}$-Cetylpyridinium Chloride- $\mathrm{KSCN}-\mathrm{CCl}_{4}$ in Aqueous System}

\author{
Shozo Shimada, Kenzo Tabuchi, ${ }^{*}$ and Masahiro Tsunooka** \\ SUNFCO, Ltd., 5-5, Nishitenma 4-chome, Kita-ku, Osaka 530, Japan \\ * Department of Industrial Chemistry, Niihama National College of Technology, \\ 7-1, Yagumo-cho, Niihama 792, Japan \\ ** Department of Applied Chemistry, Faculty of Engineering, \\ University of Osaka Prefecture, Sakai, \\ Osaka 591, Japan
}

(Received February 24, 1995)

KEY WORDS Photopolymerization / Methyl Methacrylate / N-Cetylpyridinium Chloride / Potassium Thiocyanate / Carbon Tetrachloride /

The previous paper ${ }^{1}$ reported that methyl methacrylate (MMA) was photopolymerized in aqueous system with $N$-cetylpyridinium chloride (CPC) in the presence of small quantities of $\mathrm{CCl}_{4}$, and when $\mathrm{CPC}$ was added at more than its critical micelle concentration $(\mathrm{CMC}=0.09 \mathrm{mmol} / 100 \mathrm{ml})$ photopolymerization showed characteristic features of emulsion polymerization, where CPC acted as an emulsifier as well as photosensitizer. The following photoinitiation mechanism is suggested for this photopolymerization. The CT

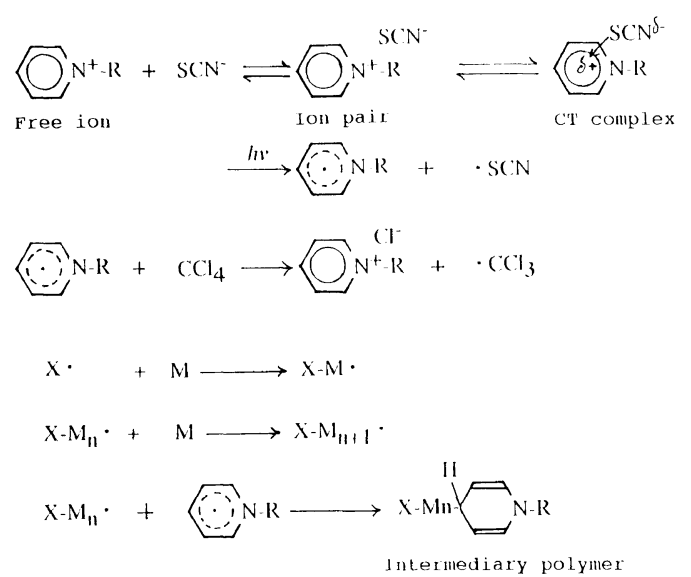

Scheme 1. Photopolymerization in the interface domain $\mathrm{R}=\mathrm{Cetyl}, \mathrm{X} \cdot=\cdot \mathrm{SCN}$ or $\cdot \mathrm{CCl}_{3}$. complex of CPC at the interface of micelles photodecomposes into $N$-cetylpyridinyl radical and $\mathrm{Cl} \cdot$, and the $\mathrm{N}$-cetylpyridinyl radical reacts with $\mathrm{CCl}_{4}$ to yield $\mathrm{CPC}$ and $\mathrm{CCl}_{3} \cdot \mathrm{Cl} \cdot$ and $\mathrm{CCl}_{3} \cdot$ initiate the polymerization of $\mathrm{MMA}$ dissolved in the micelles yielding small latex particles which grow larger and larger by the polymerization of MMA taken into the latex particles from monomer droplets. The accelerating effect of $\mathrm{KBr}$ added to this polymerization system was described in the previous paper. In this paper, $\mathrm{KSCN}$ was used instead of $\mathrm{KBr}$, since the thiocyanate ion has a greater electrodonating capacity than chloride and bromide

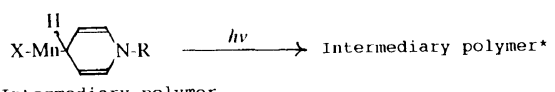

$$
\begin{aligned}
& \text { Intermediary polymer }
\end{aligned}
$$

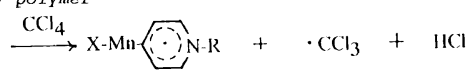

$$
\begin{aligned}
& \mathrm{X}-\mathrm{Mn}-\mathrm{CCl}_{4} \stackrel{\mathrm{fast}}{\longrightarrow} \mathrm{X}-\mathrm{Mn}-\mathrm{O}^{\mathrm{Nl}^{+}-\mathrm{R}}+\mathrm{CCl}_{3} \\
& \cdot \mathrm{CCl}_{3}+\mathrm{M} \longrightarrow \mathrm{CCl}_{3}-\mathrm{M} \text {. } \\
& \mathrm{CCl}_{3}-\mathrm{M}_{\mathrm{n}} \cdot+\mathrm{M} \longrightarrow \mathrm{CCl}_{3}-\mathrm{M}_{\mathrm{n}+1} \text {. } \\
& \mathrm{CCl}_{3}-\mathrm{M}_{\mathrm{n}} \cdot+\mathrm{CCl}_{3}-\mathrm{M}_{\mathrm{m}} \cdot \longrightarrow \mathrm{CCl}_{3}-\mathrm{M}_{\mathrm{n}+\mathrm{m}} \cdot \mathrm{CCl}_{3} \\
& \text { or } \mathrm{CCl}_{3}-\mathrm{M}_{\mathrm{n}}+\mathrm{CCl}_{3}-\mathrm{M}_{\mathrm{m}}
\end{aligned}
$$

Scheme 2. Photopolymerization in the organic bulk phase $\mathrm{R}=$ Cetyl. 
ions, ${ }^{2}$ and the thiocyano radical can effectively initiate the polymerization of $\mathrm{MMA}^{3}$ In this paper the photopolymerization of MMA in aqueous system with $\mathrm{CPC}-\mathrm{KSCN}$ in the presence of $\mathrm{CCl}_{4}$ is discussed.

\section{EXPERIMENTAL}

\section{Materials}

MMA was purified by the usual method just before use. Water was deionized. CPC of reagent grade (Tokyo Kasei Kogyo) were used without further purification. All other chemicals were of reagent grade.

\section{Polymerization}

Photopolymerizations in aqueous system were performed by the use of photochemical reaction apparatus described in detail in the previous paper. ${ }^{4}$ A $150 \mathrm{~cm}^{3}$ reaction vessel was charged with $20 \mathrm{~cm}^{3}$ of MMA solution containing $\mathrm{CCl}_{4}$ and $80 \mathrm{~cm}^{3}$ of an aqueous solution containing prescribed amounts of CPC and KSCN. The contents in the reaction vessel were bubbled by blowing pure nitrogen at constant temprature $(303 \mathrm{~K})$ and flow rate $\left(1 \mathrm{~cm}^{3} \mathrm{~s}^{-1}\right)$ through a glass filter from the bottom of the reaction vessel and irradiated by a Fuji Glass High Pressure Mercury Lamp HL-100 $(100 \mathrm{~W})$. After irradiation, the reaction mixture was poured into a large quantity of methanol and the precipitated polymer was filtered, washed with methanol, and dried in vacuo.

\section{Molecular Weight}

The molecular weight of poly(methyl methacrylate) (PMMA) was determined by gel permeation chromatography (GPC). GPC experiments were carried out on a Yanagimoto High Performance Chromatograph ModelL2000, equipped with a column Shodex A802A803, using tetrahydrofuran as the eluting solvent. The molecular weight of PMMA was estimated from the elution volume of the polymer and compared with that of standard polystyrene.

\section{RESULTS AND DISCUSSION}

The photopolymerizations of MMA with $\mathrm{CPC}$ in the presence of $\mathrm{CCl}_{4}$ were carried out by varying fed quantities of $\mathrm{KSCN}$ in an aqueous system. The conversion-time relations of the photopolymerization are shown in Figure 1. The rate of photopolymerization $\left(R_{\mathrm{p}}\right)$ was remarkably accelerated by the addition of a small quantities of $\mathrm{KSCN}$. This suggests that CT complex of $N$-cetylpyridinium thiocyanate (CPT) may be formed in sufficient amount to photoinitiate the polymerization of MMA effectively. A similar CT complex formation from the analogous pyridinium salt, $N$-benzylpyridinium thiocyanate has been already reported. ${ }^{4}$ However, when $\mathrm{KSCN}$ was added at more than one tenth the quantity of CPC, the acceleration effect was rather diminished.

Number average molecular weights $\left(M_{n}\right)$ of the polymers obtained with the varying quantities of KSCN were determined (Figure 2). The molecular weights of the obtained polymers were nearly constant at about 230000 in the range from 0.05 to $0.5 \mathrm{mmol}$ of $\mathrm{KSCN}$ added, whereas much larger below $0.01 \mathrm{mmol}$

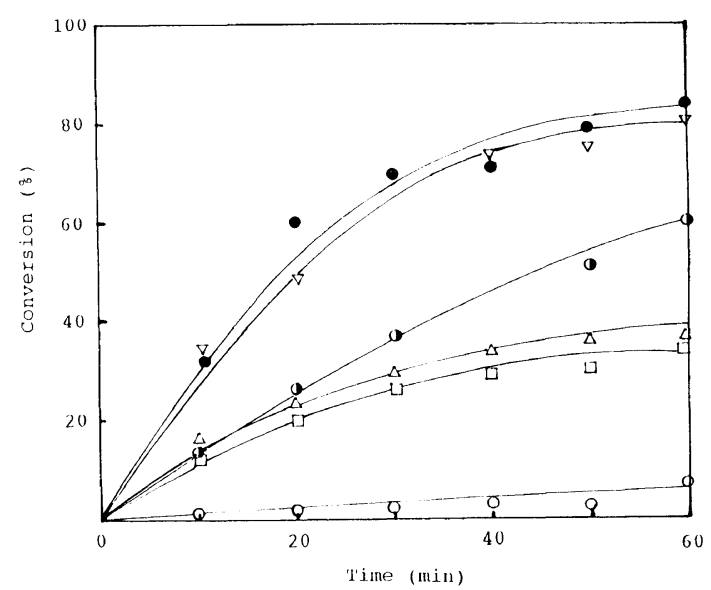

Figure 1. Effects of $\mathrm{KSCN}$ on the photopolymerization of MMA with $\mathrm{CPC}-\mathrm{CCl}_{4}$ in an aqueous system: $\mathrm{MMA}$, $20 \mathrm{~cm}^{3} ; \mathrm{H}_{2} \mathrm{O}, 80 \mathrm{~cm}^{3} ; \mathrm{CPC}, 0.5 \mathrm{mmol} ; \mathrm{CCl}_{4}, 0.5 \mathrm{mmol} ; \mathrm{N}_{2}$, $1 \mathrm{~cm}^{3} \mathrm{~s}^{-1}$; temp, $303 \mathrm{~K}$; KSCN (mmol); $\bigcirc 0.00$;, 0.01 ; ○. $0.05 ; \nabla, 0.10 ; \triangle, 0.50 ; \square, 1.0$. 


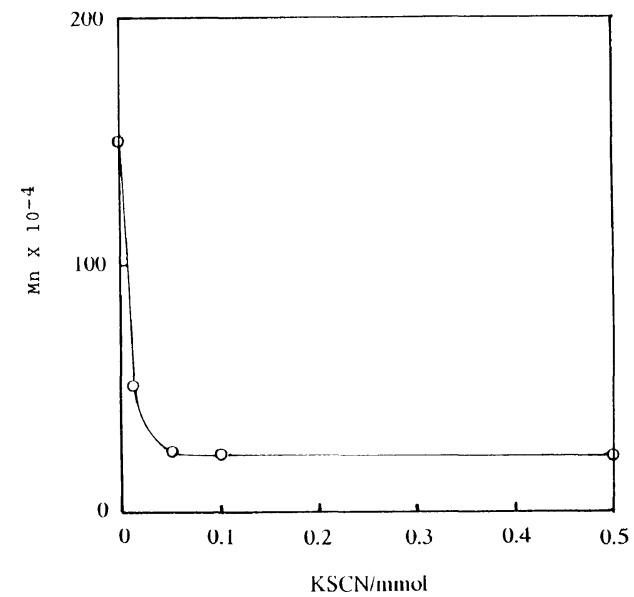

Figure 2. Relation of $M_{n}$ to added $\mathrm{KSCN}$ for the photopolymerization of MMA with $\mathrm{CPC}-\mathrm{KSCN}-\mathrm{CCl}_{4}$ : MMA, $20 \mathrm{~cm}^{3} ; \mathrm{H}_{2} \mathrm{O}, 80 \mathrm{~cm}^{3} ; \mathrm{CPC}, 0.5 \mathrm{mmol} ; \mathrm{CCl}_{4}$, $0.5 \mathrm{mmol} ; \mathrm{N}_{2}, 1 \mathrm{~cm}^{3} \mathrm{~s}^{-1}$; temp, $303 \mathrm{~K}$.

of KSCN. These facts suggest that with less than $0.05 \mathrm{mmol}$ of $\mathrm{KSCN}$ the photopolymerization proceeds in an emulsion-polymerization mode, whereas with $0.05 \mathrm{mmol}$ or more of $\mathrm{KSCN}$, the suspension-polymerization mode. In the suspension-polymerization, with the addition of $\mathrm{KSCN}$, the concentration of CPT which is more photosensitive than CPC increases in the system, but the interface area of micelles decreases by the reduction of surface active CPC which has more ionic character than CPT, and thus the accelerating effect by $\mathrm{KSCN}$ would show its maximum at a certain KSCN concentration. The molecular weight distribution during photopolymerization reaction was measured by gel permeation chromatography (GPC), and refractive index differences were plotted against elution volumes as shown in Figure 3. The polymer obtained after irradiaton for 10 minutes showed two peaks, a large one with molecular weight of $c a .20000$ and a small one with $c a .230000$. This elution pattern was reversed with increase in irradiation time, and the latter peak with 230000 led to a larger main peak after irradiation for 60 minitues. From these results, mechanisms for the photopolymerization which occurs in the following two stages are proposed. In the

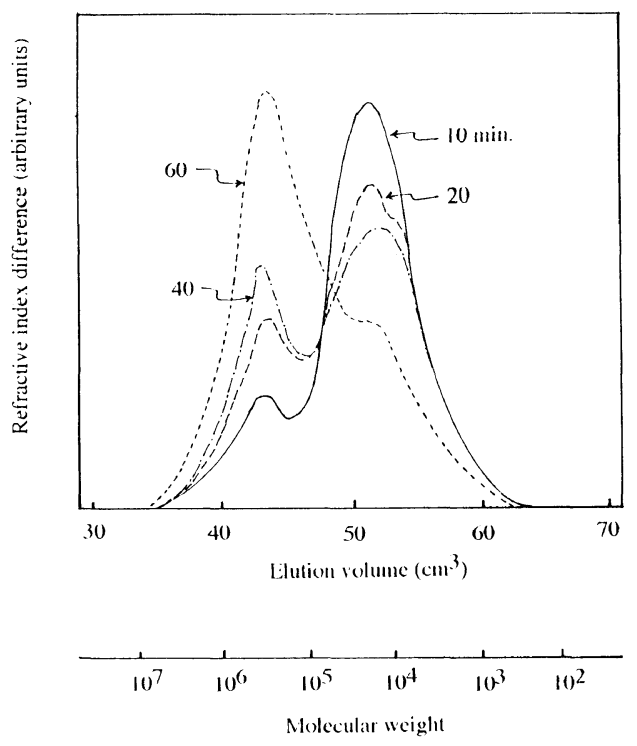

Figure 3. Gel permeation chromatograms for poly(MMA) obtained by the photopolymerization of MMA with $\mathrm{CPC}-\mathrm{KSCN}-\mathrm{CCl}_{4}$ in an aqueous system: MMA, $20 \mathrm{~cm}^{3} ; \mathrm{H}_{2} \mathrm{O}, 80 \mathrm{~cm}^{3} ; \mathrm{CPC}, 0.5 \mathrm{mmol} ; \mathrm{CCl}_{4}, 0.5 \mathrm{mmol}$; $\mathrm{KSCN}, 1.0 \mathrm{mmol} ; \mathrm{N}_{2}, 1 \mathrm{~cm}^{3} \mathrm{~s}^{-1}$; temp, $303 \mathrm{~K}$.

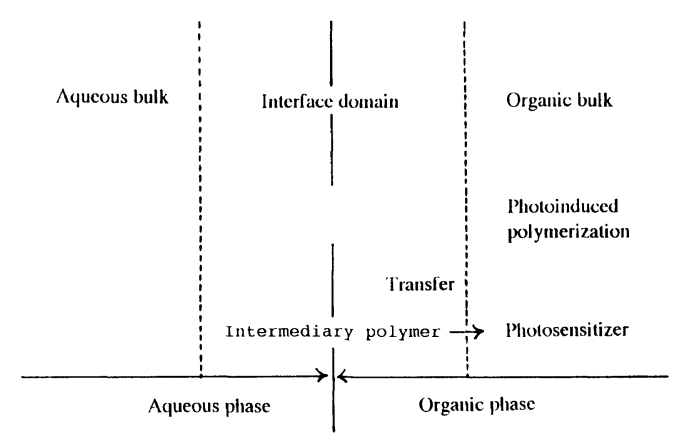

Figure 4. Domain where photopolymerization occurs.

first stage, at the interface domain as illustrated in Figure 4, photopolymerization proceeds by the mechanism shown in Scheme 1. The CT complex of $N$-cetylpyridinium thiocyanate (CPT) produced by the ion exchange of CPC with $\mathrm{SCN}^{-}$photodecomposes into thiocyano radical and $\mathrm{N}$-cetylpyridinyl radical, and the latter reacts with $\mathrm{CCl}_{4}$ to yield $\mathrm{CPC}$ and $\mathrm{CCl}_{3} \cdot$ The thiocyano radical and $\mathrm{CCl}_{3} \cdot$ initiate the polymerization of MMA, whereas the $N$ cetylpyridinyl radical contributes to termination rather than initiation due to the stability 
of the radical and yields low molecular weight. The polymer may be an intermediary polymer with 1,4-dihydropyridine residue as one of their end groups. In the second stage poly(methyl methacrylate) (PMMA) thus obtained was transferred to the organic bulk phase, and photoinduced polymerization, the mechanism of which is that proposed in Scheme 2. An analogous initiation mechanism involving the photo-excitation of 1,4-dihydropyridine derivative was reported in our earlier paper. ${ }^{5}$

Figure 5 shows the conversion-time relations for the photopolymerization of MMA at two different concentrations of $\mathrm{CCl}_{4}$ under con-

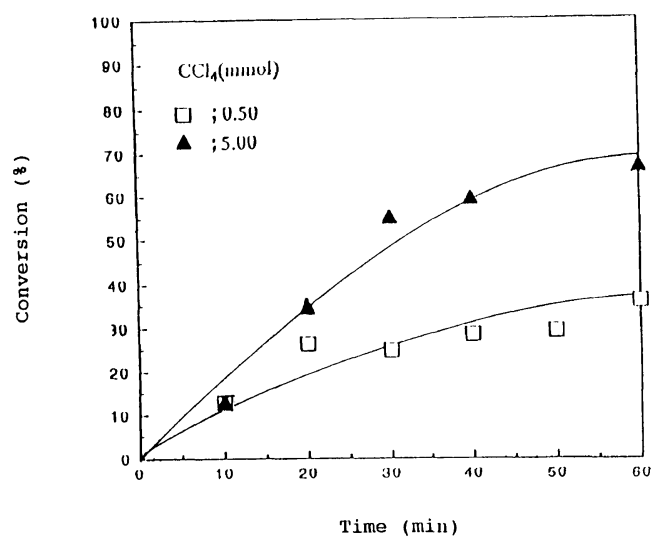

Figure 5. Effects of $\mathrm{CCl}_{4}$ concentration on the photopolymerization of MMA with $\mathrm{CPC}-\mathrm{CCl}_{4}$ in an aqueous system: MMA, $20 \mathrm{~cm}^{3} ; \mathrm{H}_{2} \mathrm{O}, 80 \mathrm{~cm}^{3} ; \mathrm{CPC}, 0.5 \mathrm{mmol}$; $\mathrm{KSCN}, 1.0 \mathrm{mmol} ; \mathrm{N}_{2}, 1 \mathrm{~cm}^{3} \mathrm{~s}^{-1}$; temp, $303 \mathrm{~K}$.

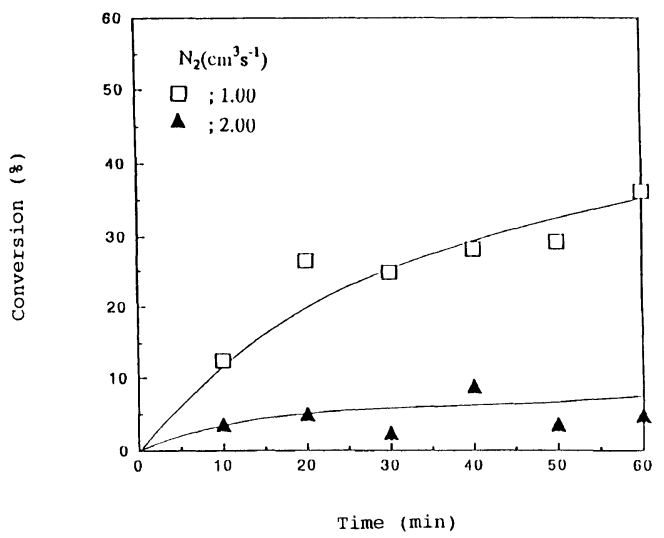

Figure 6. Effects of $\mathrm{N}_{2}$-blowing rate one the photopolymerization of MMA with $\mathrm{CPC}-\mathrm{CCl}_{4}$ in an aqueous system: MMA, $20 \mathrm{~cm}^{3} ; \mathrm{H}_{2} \mathrm{O}, 80 \mathrm{~cm}^{3}$; CPC, $0.5 \mathrm{mmol}$; $\mathrm{CCl}_{4}, 0.5 \mathrm{mmol}$; $\mathrm{KSCN}, 1.0 \mathrm{mmol}$; temp, $303 \mathrm{~K}$. stant concentrations of CPC and $\mathrm{KSCN}$. It is clear that the conversion percentage and rate of photopolymerization increased with $\mathrm{CCl}_{4}$ concentration. The molecular weights of the polymers obtained at $\mathrm{CCl}_{4}$ concentrations of $5.0 \mathrm{mmol}$ and $0.5 \mathrm{mmol}$ were $c a .160000$ and 20000 respectively. This suggests that the reactions participated by $\mathrm{CCl}_{4}$ occur mainly in the organic bulk phase rather than at the interface domain.

The rate of photopolymerization in the aqueous system was also affected by the degree of stirring which was adjusted by changing the flow rate of $\mathrm{N}_{2}$ as shown in Figure 6. The conversion and the rate of photopolymerization decreased by more intence stirring. This may be due to the remarkable increase in the interface area, where the production of intermediary polymer with low molecular weight is the main reaction as described above. These results also support the proposed mechanisms for photopolymerization. However, an attempt to characterize the end group of the intermediary polymer with low molecular weight has not been successful. In other experiments, photo-oligomerization of MMA was initiated only with $\mathrm{CPC}-\mathrm{KSCN}$ in aqueous organic two-phase system, and the structure of the obtained oligomer was elucidated and will be reported soon.

Acknowledgements. The authors are grateful to Dr. N. Sakota, Sakota Chemical Development and Research Institute Co., Ltd., for his valuable comments and helpful advice.

\section{REFERENCES}

1. S. Shimada, K. Nakagawa, and K. Tabuchi, Polym, J., 25, 1033 (1993).

2. J. F. Coetzee and J. J. Campion, J. Am. Chem. Soc., 89, 2517 (1967).

3. K. Nishihara and N. Sakota, Makromol. Chem., 165, 105 (1973).

4. K. Tabuchi and N. Sakota, Polym, J., 15, 713(1983).

5. S. Shimada, K. Nakagawa, and K. Tabuchi, Polym, J., 21, 275 (1989). 